\title{
Design of Internal Model Control Based on an Optimal Control for a Servo System
}

\author{
Hiromitsu Ogawa, Ryo Tanaka, Takahiro Murakami, and Yoshihisa Ishida \\ Meiji University School of Science and Technology, 1-1-1, Higashi-mita, Tama-ku, Kawasaki 214-8571, Japan \\ Correspondence should be addressed to Hiromitsu Ogawa; h_ogawa@meiji.ac.jp
}

Received 16 August 2014; Revised 10 February 2015; Accepted 10 February 2015

Academic Editor: Ai-Guo Wu

Copyright ( 2015 Hiromitsu Ogawa et al. This is an open access article distributed under the Creative Commons Attribution License, which permits unrestricted use, distribution, and reproduction in any medium, provided the original work is properly cited.

This paper describes a design of internal model control based on an optimal control for a servo system. The control system has the feedback based on the proposed disturbance compensator in the disturbance response. The compensator is designed to become the denominator of the transfer function without a dead time in the disturbance responses. The disturbance response of the proposed method is faster than that of the previous method.

\section{Introduction}

A PID control is one of the attractive controls for the designers and the researchers because the PID controller is simple and easy to understand sensuously. It is widely studied in the control study and used in the industrial area widely [1-5]. Although the PID control provides the benefits as described above, the parameters tuning is difficult especially for the plant with the dead time. To overcome it, Smith predictor and internal model control (IMC) have been proposed and have been studied widely to control a plant with the dead time [611]. One of the key features is to be able to design each parameter easily compared with the PID control for the plant with the dead time. IMC especially possess many advantages over the PID control, particularly in the presence of the significant process dead time. IMC simplify the control system design in a large class of industrial applications for the disturbance response. One of the simple control system design approaches utilizes an optimal control theory [12]. It is not necessary to design some controller parameters also for experienced designers and there is no steady-state error in the disturbance response. However, the parameters of its disturbance compensator are designed by trial and error in consideration of dead time. It is one of the serious issues. This is because there are various plants with the dead time in the industrial areas, such as a DC motors control, a pneumatic actuators control, and a temperature control. Generally, these plants have large dead time. In especially the DC motor control area when the system has some gears, the system has large dead time.

In this paper, we propose the design of IMC based on an optimal control for a servo system. The proposed disturbance compensator is designed to become the denominator of the closed loop transfer function without the dead time in the disturbance responses. As a result, the design of the controller is easy and its stability is improved. For this proposed method, it is not necessary to design the parameters of its disturbance compensator by trial and error like the previous method [12]. We confirm that the disturbance response of the proposed method is improved as compared with the previous method in the simulation result and the actual DC motor control result.

\section{Previous IMC Design Method}

The design method of IMC is a superior control method for the plants with the dead time. This previous method is one of the approaches to improve the steady-state error in the disturbance response [12]. Let us consider the following second-order plant with the dead time:

$$
G_{p}(s)=\frac{a}{s(s+b)} e^{-L s}
$$


where $L$ is the dead time. The following equation is discretized by the zero-order hold:

$$
G_{p}(z)=\widetilde{G}_{p}(z) z^{-d}
$$

where $d$ is the dead time and $\widetilde{G}_{p}(z)$ is a nominal plant. Then the relation between the plant output and the disturbance in the previous method is given as follows:

$$
\begin{aligned}
Y(z)=\widetilde{G}_{p}(z) z^{-d}\{ & D(z)+T(z) \\
& \left.-M(z)\left[Y(z)-\widehat{G}_{p}(z) z^{-\widehat{d}} T(z)\right]\right\},
\end{aligned}
$$

where $Y(z)$ is the plant output, $D(z)$ is the disturbance at the plant input, $\widehat{G}_{p}(z)$ is a plant model, $\widehat{d}$ is the estimated dead time, and $T(z)$ is obtained as follows:

$$
T(z)=\frac{-Q(z) Y(z)}{1-Q(z) \widehat{G}_{p}(z) z^{-\widehat{d}}} .
$$

Here, to design it simple, the controller $Q(z)$ is defined as follows:

$$
Q(z)=\frac{G_{c}(z)}{1+G_{c}(z) \widetilde{G}_{p}(z)} .
$$

The $G_{c}(z)$ is a PID controller as

$$
G_{c}(z)=K_{P}+K_{I} \frac{1}{z-1}+K_{D} \frac{1}{1+1 /(z-1)} .
$$

Here, the parameters $K_{P}, K_{I}$, and $K_{D}$ are designed by an optimal control method in this paper. Then, let us consider the following controllable and observable plant model;

$$
\begin{gathered}
\mathbf{x}(k+1)=\mathbf{A} \mathbf{x}(k)+\mathbf{B} \mathbf{u}_{\mathbf{P}}(k), \\
y(k)=\mathbf{C} \mathbf{x}(k),
\end{gathered}
$$

where $\mathbf{x}(k)$ is the state variable, $y(k)$ is the plant output, and $\mathbf{u}_{\mathbf{p}}(k)$ is the plant input. Then, to design the parameters $K_{P}$, $K_{I}$, and $K_{D}$ easily, let us consider the following augmented plant:

$$
\begin{aligned}
\mathbf{x}_{\mathbf{P}}(k+1)=\mathbf{A}_{\mathbf{P}} \mathbf{x}_{\mathbf{P}}(k)+\mathbf{B}_{\mathbf{P}} \Delta \mathbf{u}_{\mathbf{P}}(k), \\
\mathbf{e}(k)=\mathbf{C}_{\mathbf{P}} \mathbf{x}_{\mathbf{P}}(k), \\
\mathbf{x}_{\mathbf{P}}(k)=\left[\begin{array}{c}
\Delta \mathbf{x}(k) \\
\mathbf{e}(k)
\end{array}\right], \\
\mathbf{A}_{\mathbf{P}}=\left[\begin{array}{cc}
\mathbf{A} & 0 \\
-\mathbf{C} & 1
\end{array}\right], \\
\mathbf{B}_{\mathbf{P}}=\left[\begin{array}{l}
\mathbf{B} \\
0
\end{array}\right], \\
\mathbf{C}_{\mathbf{P}}=\left[\begin{array}{ll}
\mathbf{0} & 1
\end{array}\right],
\end{aligned}
$$

where $\mathbf{e}(k), \Delta \mathbf{x}(k)$, and $\Delta \mathbf{u}_{\mathbf{P}}(k)$ are described as follows:

$$
\begin{gathered}
\mathbf{e}(k)=\mathbf{r}(k)-\mathbf{y}(k), \\
\Delta \mathbf{x}(k)=\mathbf{x}(k)-\mathbf{x}(k-1), \\
\Delta \mathbf{u}_{\mathbf{P}}(k)=\mathbf{u}_{\mathbf{P}}(k)-\mathbf{u}_{\mathbf{P}}(k-1) .
\end{gathered}
$$

The performance index is used in practice for quadratic optimal control formulation as follows:

$$
J=\sum_{k=0}^{\infty}\left\{\mathbf{x}_{\mathbf{P}}^{\mathrm{T}}(k) \mathbf{Q}_{\mathbf{o}} \mathbf{x}_{\mathbf{P}}(k)+\Delta \mathbf{u}_{\mathbf{P}}^{\mathrm{T}}(k) \mathbf{R}_{\mathbf{o}} \Delta \mathbf{u}_{\mathbf{P}}(k)\right\},
$$

where $\mathbf{Q}_{\mathbf{0}}$ is a positive semidefinite real symmetric matrix and $\mathbf{R}_{\mathbf{o}}$ is a positive definite real symmetric matrix. The first term accounts for the relative importance of the state of the plant and the second term accounts for the expenditure of the energy of the control signal. Then, the following Riccati equation is solved to determine the $\Delta \mathbf{u}_{\mathbf{p}}(k)$ such that this performance index is minimized:

$$
\begin{aligned}
\mathbf{A}_{\mathbf{P}}{ }^{\mathrm{T}} \mathbf{P} \mathbf{A}_{\mathbf{P}}-\mathbf{P}-\mathbf{A}_{\mathbf{P}}{ }^{\mathrm{T}} \mathbf{P} \mathbf{B}_{\mathbf{P}}\left(\mathbf{B}_{\mathbf{P}}^{\mathrm{T}} \mathbf{P} \mathbf{B}_{\mathbf{P}}+\mathbf{R}_{\mathbf{o}}\right)^{-1} \mathbf{B}_{\mathbf{P}}{ }^{\mathrm{T}} \mathbf{P} \mathbf{A}_{\mathbf{P}} \\
+\mathbf{Q}_{\mathbf{o}}=0 .
\end{aligned}
$$

Then, the $\Delta \mathbf{u}_{\mathbf{P}}(k)$ is obtained as follows:

$$
\Delta \mathbf{u}_{\mathbf{P}}(k)=\left(\mathbf{B}_{\mathbf{P}}{ }^{\mathrm{T}} \mathbf{P} \mathbf{B}_{\mathbf{P}}+\mathbf{R}_{\mathbf{o}}\right)^{-1} \mathbf{B}_{\mathbf{P}}{ }^{\mathrm{T}} \mathbf{P} \mathbf{A}_{\mathbf{P}} \mathbf{x}_{\mathbf{P}}(k) .
$$

The $\Delta \mathbf{u}_{\mathbf{P}}(k)$ represents the input as follows:

$$
\Delta \mathbf{u}_{\mathbf{P}}(k)=\left[\begin{array}{ll}
\mathbf{F}_{1} & F_{2}
\end{array}\right]\left[\begin{array}{c}
\Delta \mathbf{x}(k) \\
\mathbf{e}(k)
\end{array}\right] .
$$

Then, the plant input is as follows:

$$
\mathbf{u}_{\mathbf{P}}(k)=\left[\begin{array}{ll}
\mathbf{F}_{1} & F_{2}
\end{array}\right]\left[\begin{array}{c}
\mathbf{x}(k) \\
\sum_{i=0}^{k} \mathbf{e}(i)
\end{array}\right] .
$$

The optimal feedback gains $\mathbf{F}_{1}=\left[\begin{array}{ll}K_{P} & K_{D}\end{array}\right]^{\mathrm{T}}$ and $F_{2}=K_{I}$ are obtained as PID parameters of $G_{c}(z)$. Then, the controller $Q(z)$ is designed by using these PID parameters. Then, the disturbance response is expressed as follows:

$$
\begin{aligned}
Y(z)= & \widetilde{G}_{p}(z) z^{-d} D(z)-\widetilde{G}_{p}(z) z^{-d} \\
& \cdot \frac{Q(z)+M(z)}{Q(z)} \frac{Q(z) Y(z)}{1-Q(z) \widehat{G}_{p}(z) z^{-d}} \\
= & \widetilde{G}_{p}(z) z^{-d} D(z)-\widetilde{G}_{p}(z) z^{-d} \\
& \cdot[Q(z)+M(z)] \frac{Y(z)}{1-Q(z) \widehat{G}_{p}(z) z^{-d}}, \\
Y(z)= & \frac{1-Q(z) \widehat{G}(z) z^{-\widehat{d}}}{1-Q(z) \widehat{G}(z) z^{-\widehat{d}}+\widetilde{G}_{p}(z) z^{-d}[Q(z)+M(z)]} \\
& \cdot \widetilde{G}_{p}(z) z^{-d} D(z) .
\end{aligned}
$$




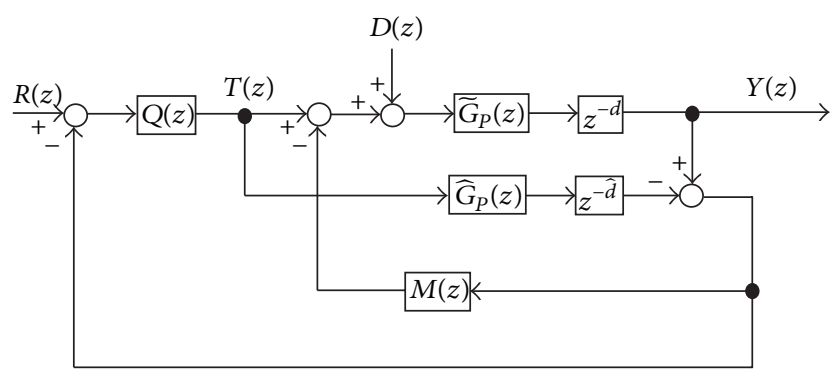

FIGURE 1: A block diagram of the proposed method.

To consider it easily, we assume that

$$
\widehat{G}_{p}(z)=\widetilde{G}_{p}(z), \quad \widehat{d}=d .
$$

Then the following disturbance response is obtained as follows:

$$
Y(z)=\frac{1-Q(z) \widetilde{G}_{p}(z) z^{-d}}{1+M(z) \widetilde{G}_{p}(z) z^{-d}} \widetilde{G}_{p}(z) z^{-d} D(z) .
$$

In this previous method [12], the $M(z)$ is a PID controller as follows:

$$
M(z)=K_{P M}+K_{I M} \frac{1}{z-1}+K_{D M} \frac{1}{1+1 /(z-1)} .
$$

These parameters, $K_{P M}, K_{I M}$, and $K_{D M}$, are designed on a trial basis.

\section{Proposed IMC Design Method}

Although the previous method does not have the steadystate error in the disturbance response at the plant input, the denominator of the disturbance response contains the dead time. In addition, $M(z)$ is designed by trial and error. However, it is difficult to design $M(z)$. To overcome this problem, we propose the design of the disturbance compensator $M(z)$ as follows:

$$
M(z)=\frac{1}{1-\widetilde{G}_{p}(z)\left(z^{-d}-1\right)} .
$$

Then let us substitute (19) into (18) as follows:

$$
\begin{aligned}
Y(z) & =\frac{\left(1+G_{c}(z) \widetilde{G}_{p}(z)\left(1-z^{-d}\right)\right) /\left(1+G_{c}(z) \widetilde{G}_{p}(z)\right)}{1+\left(1 /\left(1-\widetilde{G}_{p}(z)\left(z^{-d}-1\right)\right)\right) \widetilde{G}_{p}(z) z^{-d}} \widetilde{G}_{p}(z) z^{-d} D(z) \\
& =\frac{\left(\left(1+G_{c}(z) \widetilde{G}_{p}(z)\left(1-z^{-d}\right)\right) /\left(1+G_{c}(z) \widetilde{G}_{p}(z)\right)\right)\left(1-\widetilde{G}_{p}(z)\left(z^{-d}-1\right)\right)}{\left(1+\left(1 /\left(1-\widetilde{G}_{p}(z)\left(z^{-d}-1\right)\right)\right) \widetilde{G}_{p}(z) z^{-d}\right)\left(1-\widetilde{G}_{p}(z)\left(z^{-d}-1\right)\right)} \widetilde{G}_{p}(z) z^{-d} D(z) \\
& =\frac{\left(\left(1+G_{c}(z) \widetilde{G}_{p}(z)\left(1-z^{-d}\right)\right) /\left(1+G_{c}(z) \widetilde{G}_{p}(z)\right)\right)\left(1-\widetilde{G}_{p}(z)\left(z^{-d}-1\right)\right)}{\left(1-\widetilde{G}_{p}(z)\left(z^{-d}-1\right)+\widetilde{G}_{p}(z) z^{-d}\right)} \widetilde{G}_{p}(z) z^{-d} D(z) \\
& =\frac{\left(\left(1+G_{c}(z) \widetilde{G}_{p}(z)\left(1-z^{-d}\right)\right) /\left(1+G_{c}(z) \widetilde{G}_{p}(z)\right)\right)\left(1-\widetilde{G}_{p}(z)\left(z^{-d}-1\right)\right)}{1+\widetilde{G}_{p}(z)} \widetilde{G}_{p}(z) z^{-d} D(z) .
\end{aligned}
$$

Since the denominator of the disturbance response does not contain the dead time, the design of $M(z)$ is easier than the previous method. The proposed method can control the plant as shown in Figure 1.

\section{Simulation Result}

This simulation shows the effect for the dead time. Consider the following second-order plant:

$$
G_{P}(s)=\frac{a}{s(s+b)} e^{-L s} .
$$

In this simulation, we assume that the plant is a DC motor. $a$ is equal to 1 , and $b$ is also equal to 1 . Then, it is discretized by the zero-order hold. The sampling time is 10 (ms). The set point is 1 (rad) and the disturbance in the plant input is -0.05 at 5 (s). The controller $G_{c}(z)$ is designed by the optimal control method $\left(\mathbf{Q}_{\mathbf{o}} / \mathbf{R}_{\mathbf{o}}=1\right)$. The feedback gains $\mathbf{F}_{1}$ and $F_{2}$ are
$\mathbf{F}_{1}=\left[\begin{array}{ll}42.45 & 8.18\end{array}\right]$ and $F_{2}=0.96$, respectively. Figures 2, 3, and 4 show the simulation results for the plant with the dead time, $L$, are 1 (s), 10 (s), and 20 (s), respectively. From Figures 2 and 3, the output of the proposed method converges faster than that of the previous method. Moreover, from Figure 4, the proposed method can control the plant output, and the previous method cannot control it, because the denominator of the proposed method does not contain the dead time, whereas the previous method contains it.

\section{Actual DC Motor Control Result}

An electric motor has been widely applied in the industry such as automobile, robot, and transport equipment. In these days especially, a motor control is one of the key technologies in a hybrid automobile and electrical automobile. In this study, we verify that the proposed method can be applied in a motor. Figures 5 and 6 show the control system overview 


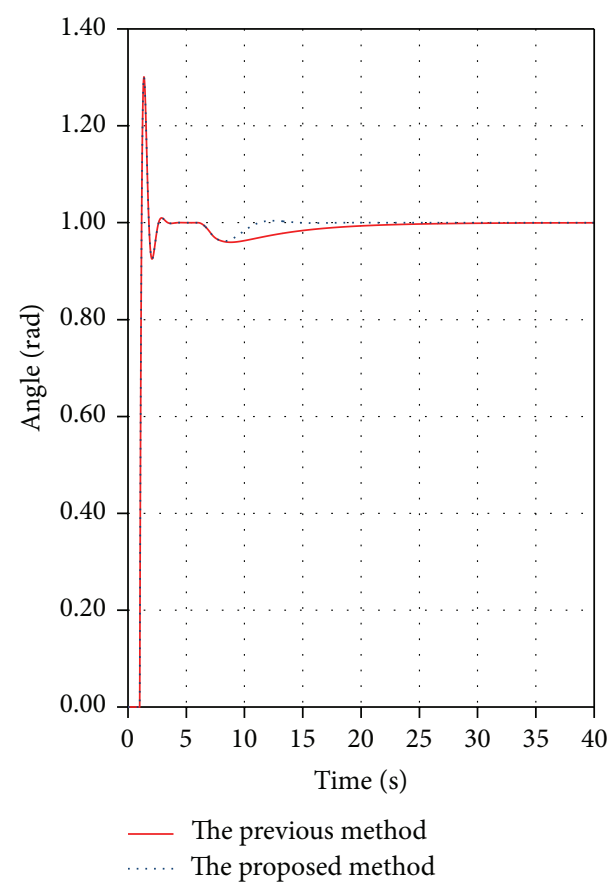

FIgURE 2: Simulation results for the plant with the dead time 1 (s).

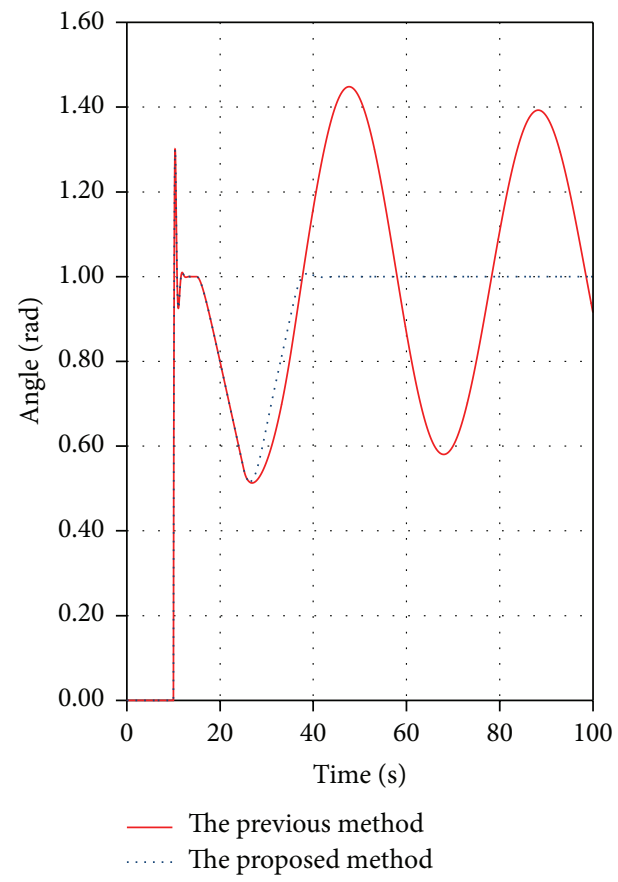

FIGURE 3: Simulation results for the plant with the dead time 10 (s).

and the DC motor control system as an image, respectively. The DC motor is made by PID Corporation (PID-QET ii). The D/A convertor resolution is 12 bits. The encoder resolution is $1.534 \times 10^{3}$ ( $\mathrm{rad} /$ count). The motor includes an inertial load disk with a radius of $0.0248(\mathrm{~m})$ and a weight of $0.068(\mathrm{~kg})$. The sampling time is $1(\mathrm{~ms})$. The set point is $1.570(\mathrm{rad})$ and the input-side disturbance is $-0.1(\mathrm{~V})$ at

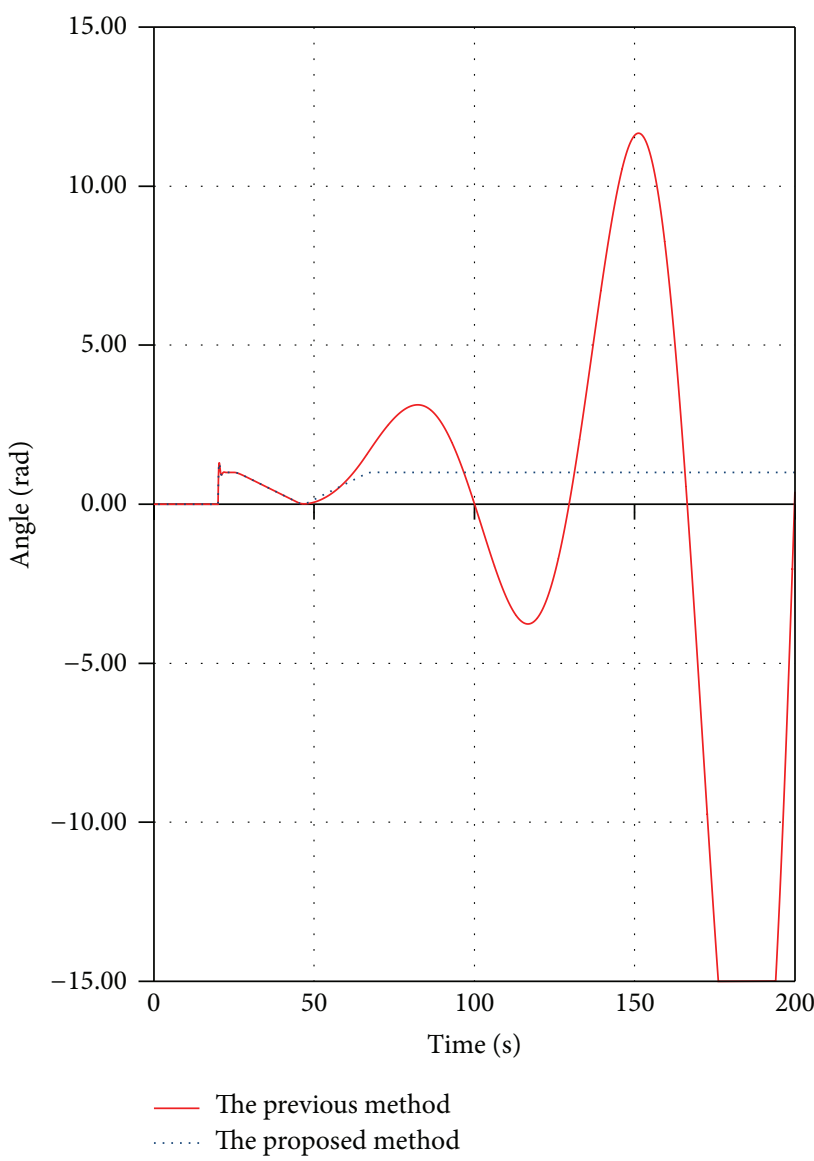

FIGURE 4: Simulation results for the plant with the dead time 20 (s).

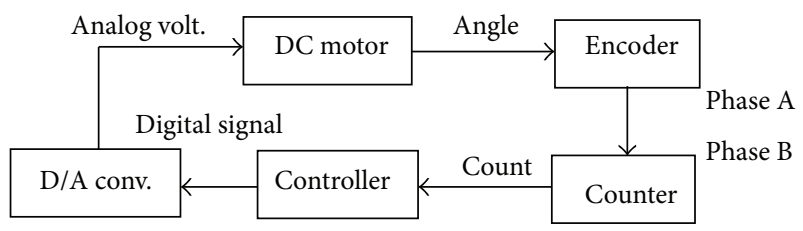

FIGURE 5: The motor control system.

10 (s). The controller $G_{c}(z)$ is designed by the optimal control method $\left(\mathbf{Q}_{\mathbf{0}} / \mathbf{R}_{\mathbf{o}}=1\right)$. The plant transfer function $G_{p}(s)$ and the setup of each parameter $a, b, \mathbf{F}_{1}$, and $F_{2}$ are set in the same manner as in simulation setups. Figure 7 shows the actual DC motor control results. The proposed method convergence in the disturbance response is faster than that of the previous method. This result also indicates that the proposed method can be applied to actual DC motors.

\section{Conclusion}

In this paper, we have proposed the design of the internal model control based on an optimal control for a servo system. The design of the disturbance compensator has been proposed, and the denominator does not contain the dead time. From the simulation results, we have confirmed that the disturbance response of the proposed method is improved as 


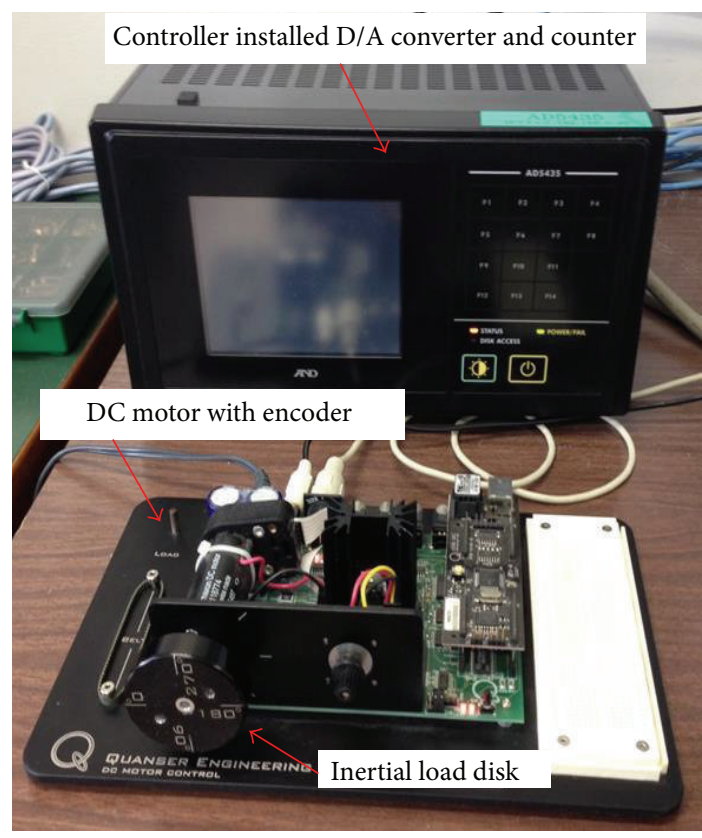

FIGURE 6: The picture of the control system.

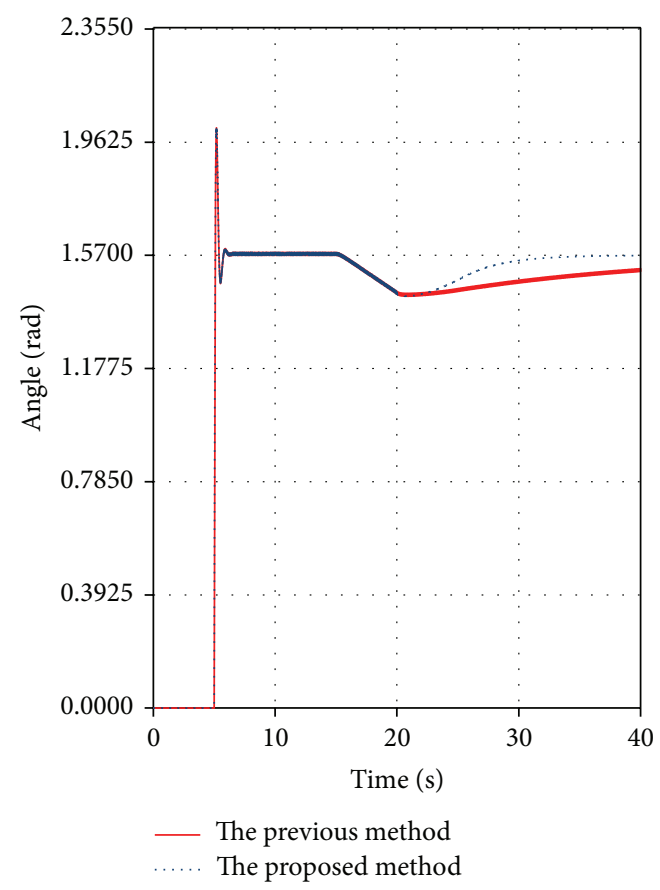

FIGURE 7: Experimental results for an actual DC motor.

compared with the previous method in the simulation results and the actual DC motor control result.

\section{Conflict of Interests}

The authors declare that there is no conflict of interests regarding the publication of this paper.

\section{References}

[1] M. Yukitomo, T. Shigemasa, Y. Baba, and F. Kojima, "A two degrees of freedom PID control system, its features and applications," in Proceedings of the 5th Asian Control Conference, vol. 1, pp. 456-459, July 2004.

[2] P. R. Ouyang, V. Pano, and T. Dam, "PID contour tracking control in position domain," in Proceedings of the 21st IEEE International Symposium on Industrial Electronics, pp. 1297-1302, May 2012.

[3] C. Huang, Y. Bai, and X. Li, "Simulation for a class of networked cascade control systems by PID control," in Proceedings of the International Conference on Networking, Sensing and Control (ICNSC '10), pp. 458-463, April 2010.

[4] J. Hao, W. Zhang, and M. Ling, "Research on PID control algorithm of BP network based on two axis digital control turntable," in Proceedings of the 10th IEEE International Conference on Computer and Information Technology (CIT '10), pp. 2233-2238, IEEE, Bradford, UK, July 2010.

[5] A. Shi, M. Yan, J. Li, W. Xu, and Y. Shi, "The research of FUZZY PID control application in DC motor of automatic doors," in Proceedings of the International Conference on Electrical and Control Engineering, pp. 1354-1358, September 2011.

[6] Z. Ming-guang, W. Peng, and W. Zhao-gang, "Study on fuzzy self-tuning PID internal model control algorithm and Its application," in Proceedings of the International Conference on Computational Intelligence and Security Workshops (CIS '07), pp. 31-34, December 2007.

[7] T.-L. Chia and I. Lefkowitz, "Internal model-based control for integrating processes," ISA Transactions, vol. 49, no. 4, pp. 519$527,2010$.

[8] X. Shao, J. Zhang, Z. Zhao, and X. Wen, "Adaptive internal model control of permanent magnet synchronous motor drive system," in Proceedings of the 8th International Conference on Electrical Machines nd Systems (ICEMS '05), vol. 3, pp. 18431846, 2005.

[9] S. Li and H. Gu, "Fuzzy adaptive internal model control schemes for PMSM speed-regulation system," IEEE Transactions on Industrial Informatics, vol. 8, no. 4, pp. 767-779, 2012.

[10] H. Yanwei and W. Dengguo, "Nonlinear internal model control with inverse model based on extreme learning machine," in Proceedings of the International Conference on Electric Information and Control Engineering (ICEICE '11), pp. 2391-2395, April 2011.

[11] Z. Yanhong and Z. Zhongqiao, "Based on inverse system of internal model control," in Proceedings of the International Conference on Computer Application and System Modeling (ICCASM '10), vol. 14, pp. 19-21, Taiyuan, China, October 2010.

[12] H. Ogawa, R. Tanaka, T. Murakami, and Y. Ishida, "Improved internal model control based on optimal control for servo system with dead time," in Proceedings of the IEEE 10th International Conference on Power Electronics and Drive Systems (PEDS '13), pp. 731-734, April 2013. 

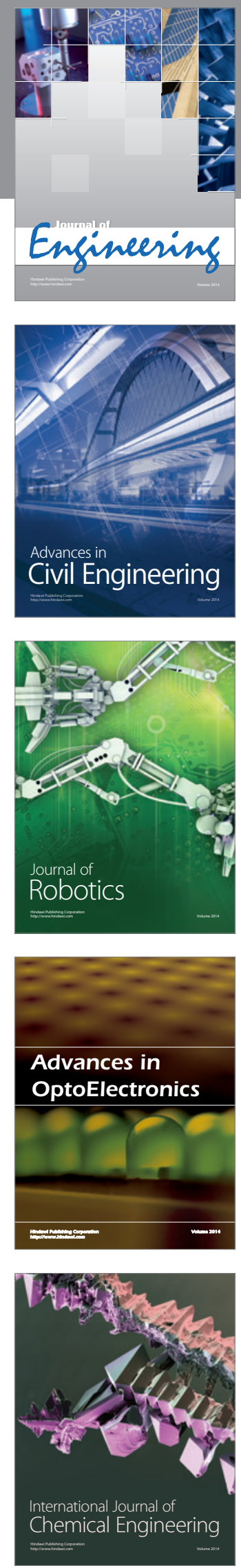

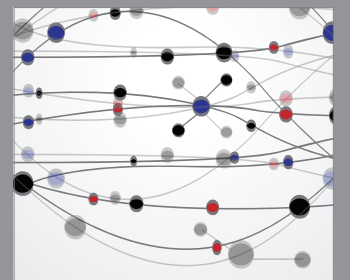

The Scientific World Journal
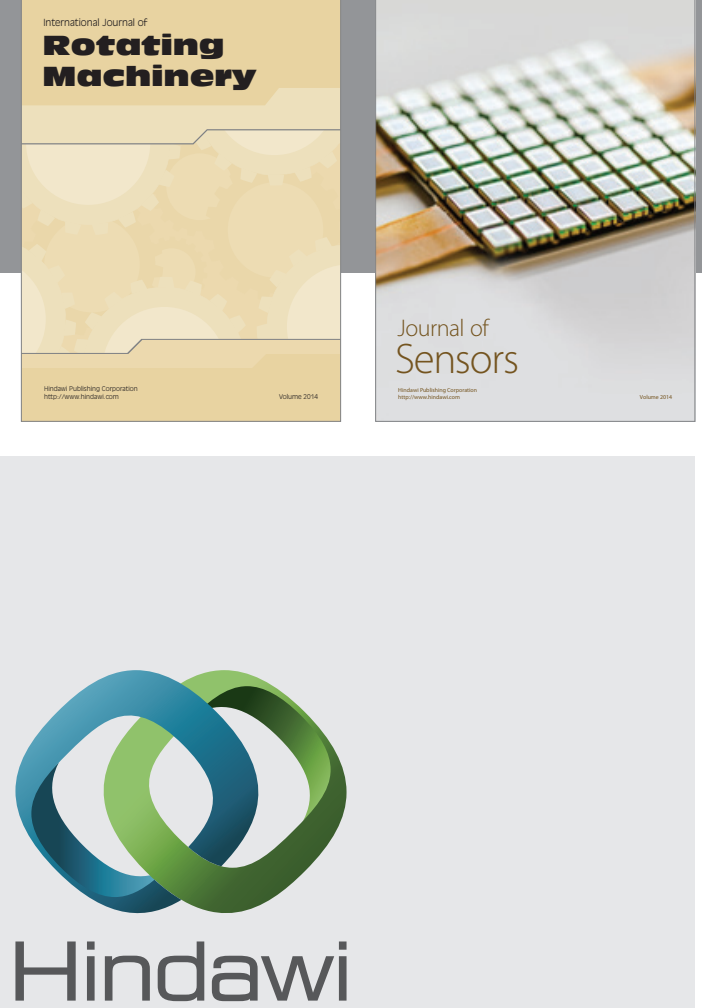

Submit your manuscripts at http://www.hindawi.com
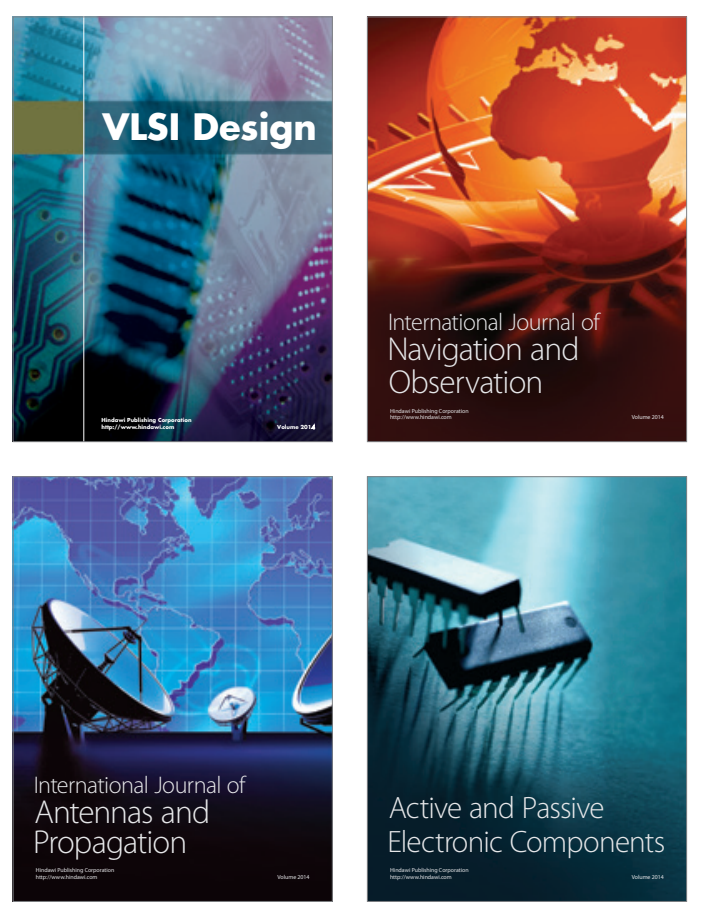
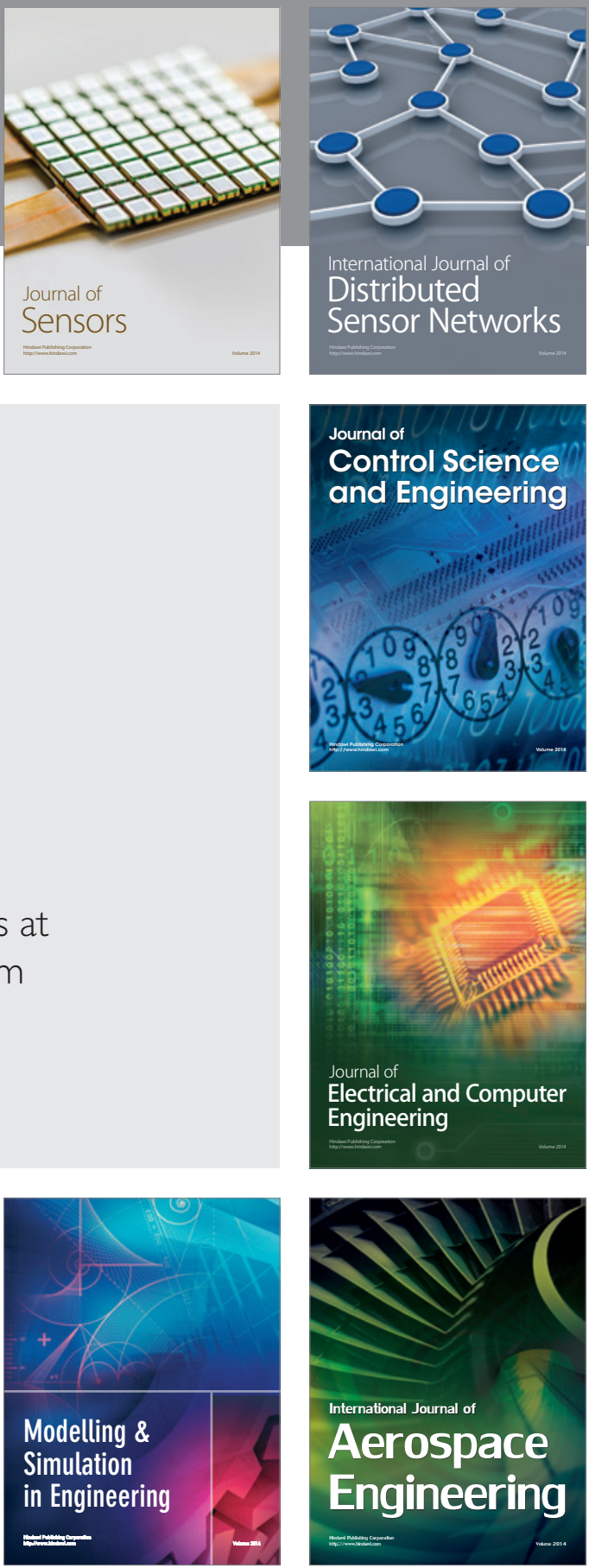

Journal of

Control Science

and Engineering
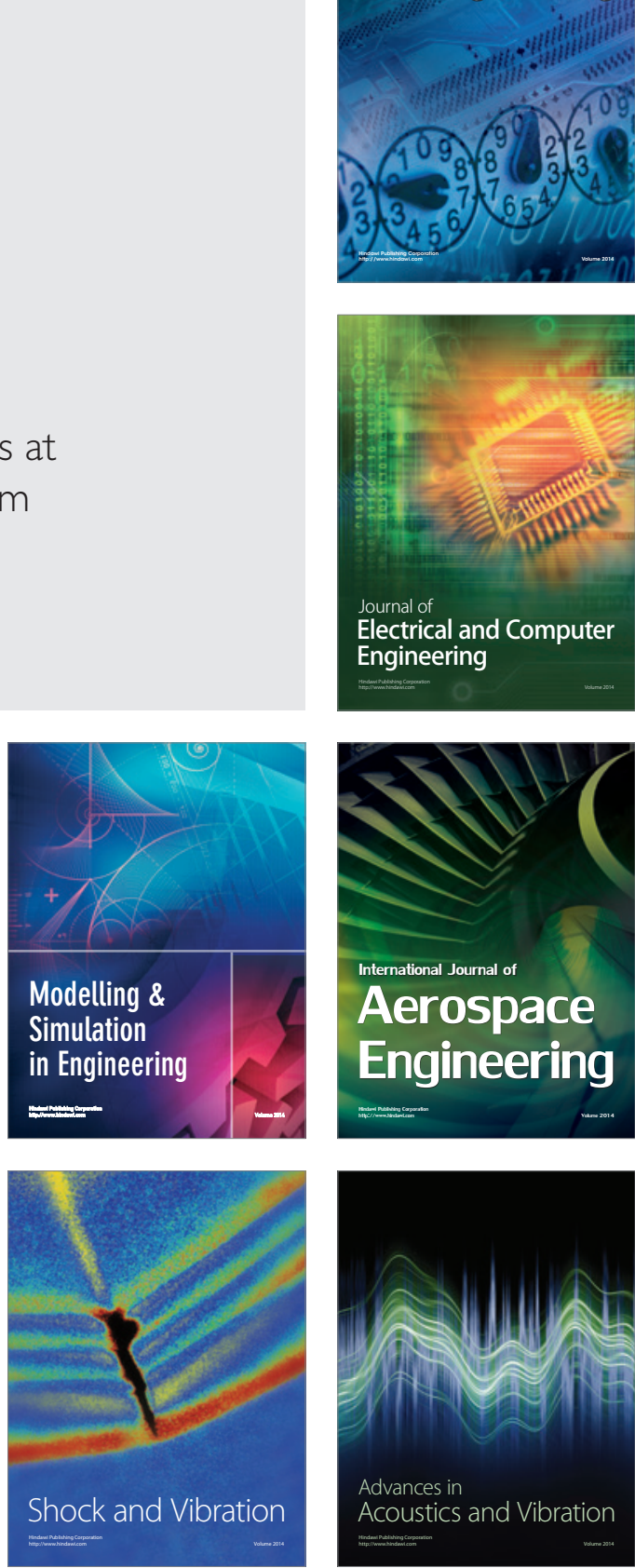